\title{
EFFECT OF THE SUBSTRATE CRYSTALLINE ORIENTATION ON THE SURFACE MORPHOLOGY AND BORON INCORPORATION INTO EPITAXIAL DIAMOND LAYERS
}

\author{
Jan VOVES ${ }^{1}$, Alexandr POŠTA 1 , Marina DAVYDOVA², Alexandr LAPOSA ${ }^{1}$, Vojtěch POVOLNÝ1, \\ Pavel HAZDRA ${ }^{1}$, Nicolas LAMBERT ${ }^{1,2}$, Silvia SEDLAKOVÁ ${ }^{2}$, Vincent MORTET ${ }^{2,3}$ \\ ${ }^{1}$ Faculty of Electrical Engineering, Czech Technical University in Prague, Prague, Czech Republic, EU, \\ voves@fel.cvut.cz \\ ${ }^{2}$ Institute of Physics of the Czech Academy of Sciences, Prague 8, Czech Republic, EU, mortet@fzu.cz \\ ${ }^{3}$ Faculty of Biomedical Engineering, Czech Technical University in Prague, Kladno, Czech Republic, EU
}

https://doi.org/10.37904/nanocon.2020.3683

\begin{abstract}
Epitaxial growth of diamond is critically important for the fabrication of diamond-based electronic devices. The emerging study of the epitaxial diamond growth on the (113) vicinal surfaces evidences highly needed high growth rates and low structural defects concentrations with both $\mathrm{p}$ - and $\mathrm{n}$-type doping. In this work, we compare the morphology and dopant concentration incorporation of heavily boron-doped (113) epitaxial diamond layers with conventionally studied (100) and (111) epitaxial layers. Epitaxial layers were grown using resonance cavity Microwave Plasma Enhanced Chemical Vapor Deposition (MWPECVD) system. The surface morphology of epitaxial layers was studied by optical microscopy and atomic force microscopy, whereas the boron incorporation homogeneity was determined by Raman spectroscopy mapping. Heavily boron-doped (113) epitaxial diamond layers can be grown at a high growth rate with a smooth surface, without pyramidal hillocks or non-epitaxial crystallite defects, and with homogeneous boron concentration. These results confirm that epitaxial diamond growth on (113) vicinal surfaces is a promising solution for the development and fabrication of diamond-based electronic devices.
\end{abstract}

Keywords: Diamond, epitaxy, defects, boron doping, Raman mapping

\section{INTRODUCTION}

In recent years, modern power electronics have been exploiting new semiconductor materials with a wide bandgap such as gallium nitride or silicon carbide for improving the blocking capability, decreasing of dimensions, and increasing the switching speed of power devices [1]. Due to its outstanding physical parameters, diamond is the best wide-bandgap materials for power electronic applications. However, the preparation of epitaxy layers with a low density of defects and homogenous doping is still under development $[2,3]$. The use of $(113)$ oriented substrates for the epitaxial growth shows promising results with high boron incorporation efficiency, smooth and almost defect-free surface [4]. The Raman spectroscopy has been demonstrated as a simple and non-destructive characterization method to determine the atomic boron concentration as well as to monitor the strain connected with the presence of defects [5-7]. The increasing boron concentration red-shifts the diamond's zone-center phonon peak located at $1332 \mathrm{~cm}^{-1}$ to lower values and lower its relative intensity with respect to the asymmetric band centered around $1200 \mathrm{~cm}^{-1}$ attributed to a maximum of the phonon density of states. Another characteristic wide band centered around $500 \mathrm{~cm}^{-1}$ shows an almost linear Raman downshift with increasing boron concentration [7-9]. The inner strain in the epitaxy layer influences both the main peak and second asymmetric band [10]. In this work, we investigate and 
compare the surface morphology, and the spatial distribution of boron atoms in homo-epitaxial diamond layers grown on (100), (111), and (113) oriented substrates.

\section{EXPERIMENTAL}

Three epitaxial diamond layers with (111), (100), and (113) orientations were grown on polished diamond substrates using a $1.5 \mathrm{~kW}$ resonance cavity Microwave Plasma Enhanced Chemical Vapor Deposition (MWPECVD) system AX5010, Seki Diamond Systems. Prior diamond growth, all substrates were cleaned using the same multi steps procedure as follow: (i) cleaning in hot $\mathrm{H} 2 \mathrm{SO} 4+\mathrm{KNO} 3$ during 10 min, (ii) rinsing in deionized water in ultrasound bath for $10 \mathrm{~min}$ two times, (iil) cleaning in acetone with ultrasound for $10 \mathrm{~min}$, (iv) cleaning in isopropyl alcohol with ultrasound for $10 \mathrm{~min}$ and finally the substrate is expose at high temperature to pure hydrogen microwave plasma for $10 \mathrm{~min}$ before the introduction of the precursor gases and the diamond growth. This cleaning process removes efficiently all surface contaminant as observed by $\mathrm{X}$ Ray Photoelectron Spectroscopy (not shown here). The growth parameters for the different epitaxial layers are summarized in Table 1. The surface morphology of epitaxial layers was observed using an optical microscope Olympus BX60 and an atomic force microscope (AFM) NT-MDT Ntegra Prima. The boron incorporation homogeneity was studied by Raman surface mapping with a Raman Spectrometer Renishaw inVia Qontor equipped with a 532nm laser. The shift of the Raman wideband centered around $500 \mathrm{~cm}^{-1}$ was used to map the boron concentration, whereas the exact boron concentration was determined from the width of the diamond's zone-center phonon peak $\left(1332 \mathrm{~cm}^{-1}\right)$ using the method described in [11] (see Figure 1).

Table 1 Diamond samples with different surface plane orientations, boron/carbon gas precursor concentration ratios, and epitaxy growth times

\begin{tabular}{|c|c|c|c|c|c|c|}
\hline $\begin{array}{c}\text { Crystalline } \\
\text { orientation }\end{array}$ & $\begin{array}{c}\text { Microwave } \\
\text { power (W) }\end{array}$ & $\begin{array}{c}\text { Pressure } \\
\text { (mbar) }\end{array}$ & $\begin{array}{c}\text { Methane } \\
\text { concentr. (\%) }\end{array}$ & $\begin{array}{c}\text { B/C ratio } \\
(\mathbf{p p m})\end{array}$ & $\begin{array}{c}\text { Boron } \\
\text { concentr. }\end{array}$ & $\begin{array}{c}\text { Deposition } \\
\text { rate }(\mu \mathrm{m} / \mathbf{h})\end{array}$ \\
\hline$(111)$ & 575 & 60 & $0.1 \%$ & 10,000 & $5.3 \times 10^{20}$ & $0.9-1$ \\
\hline$(100)$ & 700 & 100 & $1 \%$ & 4,000 & $1.3-10 \times 10^{20}$ & $1.8-2$ \\
\hline$(113)$ & 700 & 100 & $1 \%$ & 2,000 & $7.2 \times 10^{20}$ & $3-5$ \\
\hline
\end{tabular}

\section{RESULTS AND DISCUSSION}

All diamond samples were inspected by optical microscopy, and characteristic surface defects were further inspected by AFM and Raman spectroscopy.

\section{1. (111) oriented homo-epitaxial boron-doped diamond}

The (111) oriented homo-epitaxial diamond layer is optically very uniform over its $2 \times 2 \mathrm{~mm}^{2}$ surface with only one line defect trough (see Figure 2a). The AFM microscopy outside of the line defect shows on an inhomogeneous surface (Figure $\mathbf{2 b}$ ) with approx. $100 \mathrm{~nm}$ deep and $1 \mu \mathrm{m}$ wide trenches that were exhibiting a stochastic shape resulting in a root-mean-squared (RMS) roughness of $50 \mathrm{~nm}$. Raman spectra recorded on randomly selected points are nearly identical (see Figure 1 left) and correspond to the heavily boron-doped diamond with a metallic conductivity and the boron concentration of $\sim 5.3 \times 10^{20} \mathrm{~cm}^{-3}$, which was established from the unperturbed width of the diamond's zone-center phonon (ZCP) peak. The map of the wide Raman peak centered around $500 \mathrm{~cm}^{-1}$ measured in the region including the line defect is homogeneous (Figure 2c). This confirms the results of the Raman point-by-point measurement and shows on very good homogeneity of boron incorporation with relatively small variations associated with the line defect. 


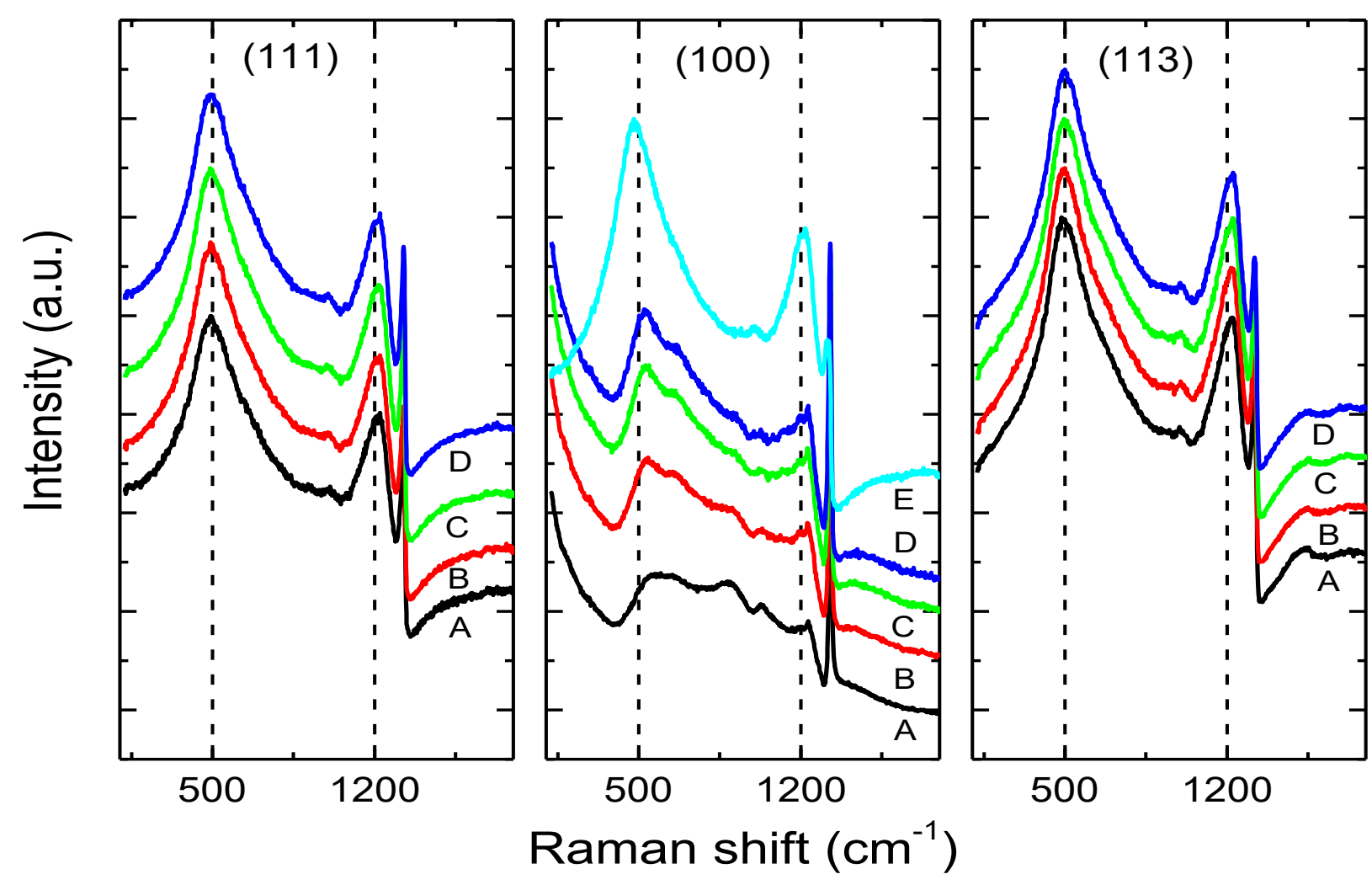

Figure 1 The Raman spectra of the homo-epitaxial boron-doped diamond layers grown on (111), (100), and (113) oriented substrates measured in the different points shown in Figures $2 \mathrm{c}, 3 \mathrm{c}$, and $4 \mathrm{c}$, respectively

\section{2. (100) oriented homo-epitaxial boron-doped diamond}

Optical microscopy observation (see Figures $\mathbf{3} \mathbf{a}$ and $\mathbf{3 b}$ ) shows that this layer is covered by large rectangular based pyramidal hillocks with approx. 100x100 $\mu \mathrm{m}$ base and $100 \mathrm{~nm}$ height. The map of one hillock, including its vicinity (Figure 3c), shows on a large dispersion of the $500 \mathrm{~cm}^{-1}$ Raman peak, which is characteristic for inhomogeneous boron incorporation inside the hillock and its vicinity. The analysis of the zone-center-phonon line at different measurement points (Figure 1 center) shows that the boron concentration varies from $1.3 \times 10^{20}$ to $10^{21} \mathrm{~cm}^{-3}$ and shows a minimal value on the top of the hillock.

\section{3. (113) oriented homo-epitaxial boron-doped diamond}

Apart from a few linear defects, the optical microscopy observation (see Figure 4a) demonstrates that the surface of the (113) oriented homo-epitaxial boron-doped diamond layer is flat and homogeneous. AFM inspection (see Figure 4b) confirms a very smooth surface with the RMS surface roughness of about $1 \mathrm{~nm}$. The Raman map of the $500 \mathrm{~cm}^{-1}$ peaks, which was measured in the region including the line defect (see Figure 4c), indicates a very good boron homogeneity through the whole sample and a negligible dispersion along the line of the defect (Figure 1 center). The boron concentration determined by the analysis of the diamond ZCP peak is approximately $7.2 \times 10^{20} \mathrm{~cm}^{-3}$. This high boron concentration gives evidence that, during diamond growth on (113) surface, boron embeds with a high boron incorporation efficiency (BIE), which reaches approx. $2 \%$. This is a substantially higher value compared to the layers grown on the (100) surface $(0.14 \%<\mathrm{BIE}<1 \%)$ and $(111)$ surfaces $(\mathrm{BIE}=0.28 \%)$. 


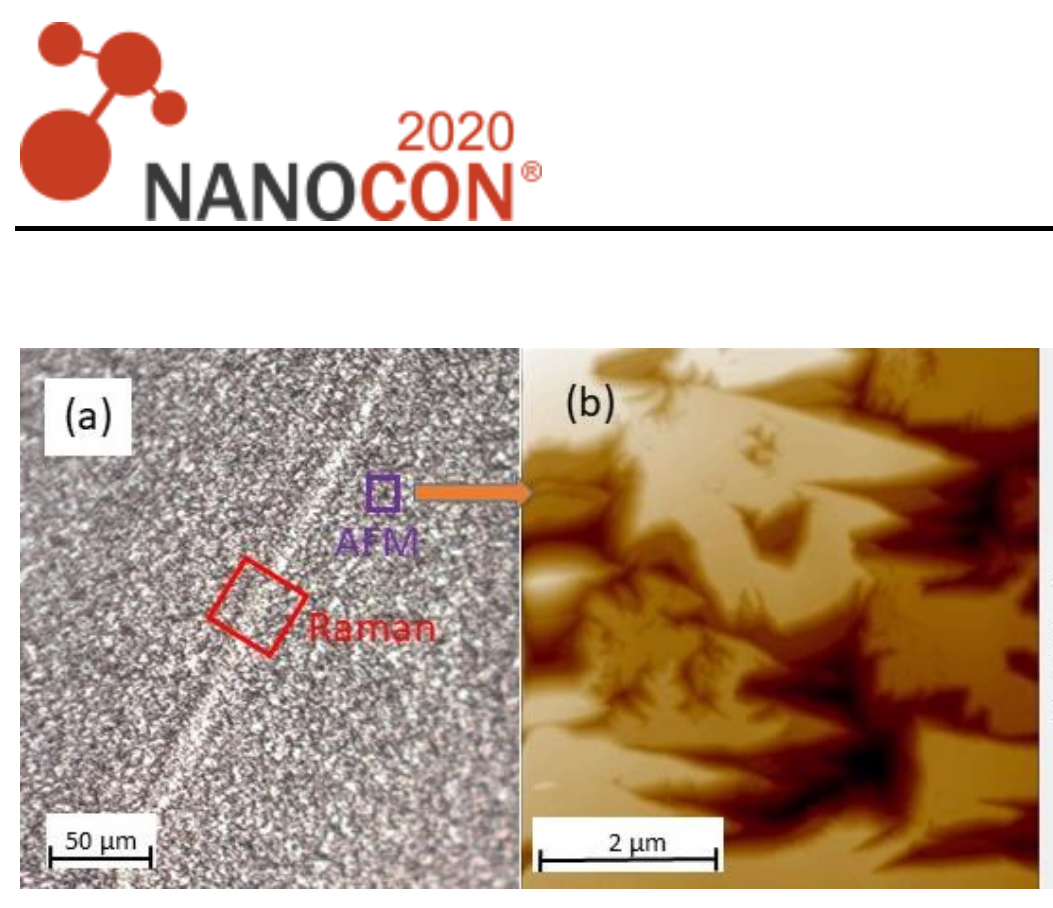

Figure 2 (a) Optical microscopy image of the (111) - oriented boron-doped diamond surface;

(b) AFM image of the diamond's surface of the area indicated by the violet square on the optical microscopy image; (c) Raman $500 \mathrm{~cm}^{-1}$ peak map of the area indicated by the red square in the optical microscopy image
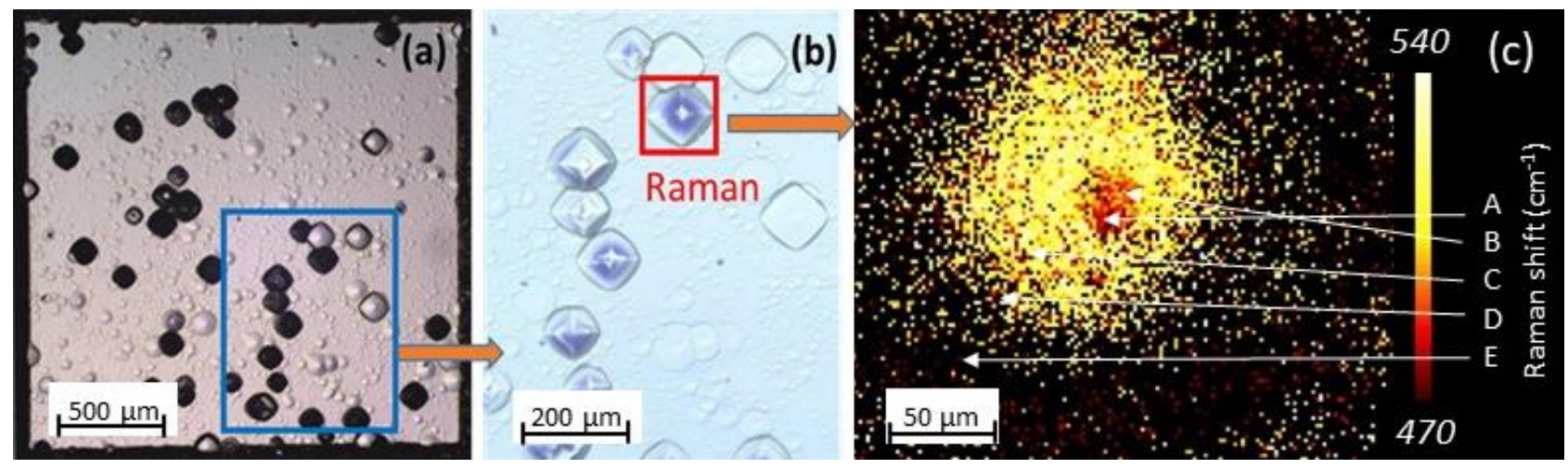

Figure 3 Large view (a) and magnified (b) of the area in the blue box optical microscopy images of the (100) - oriented boron-doped diamond surface; (c) Raman $500 \mathrm{~cm}^{-1}$ peak map of the area indicated by the red square in the optical microscopy image
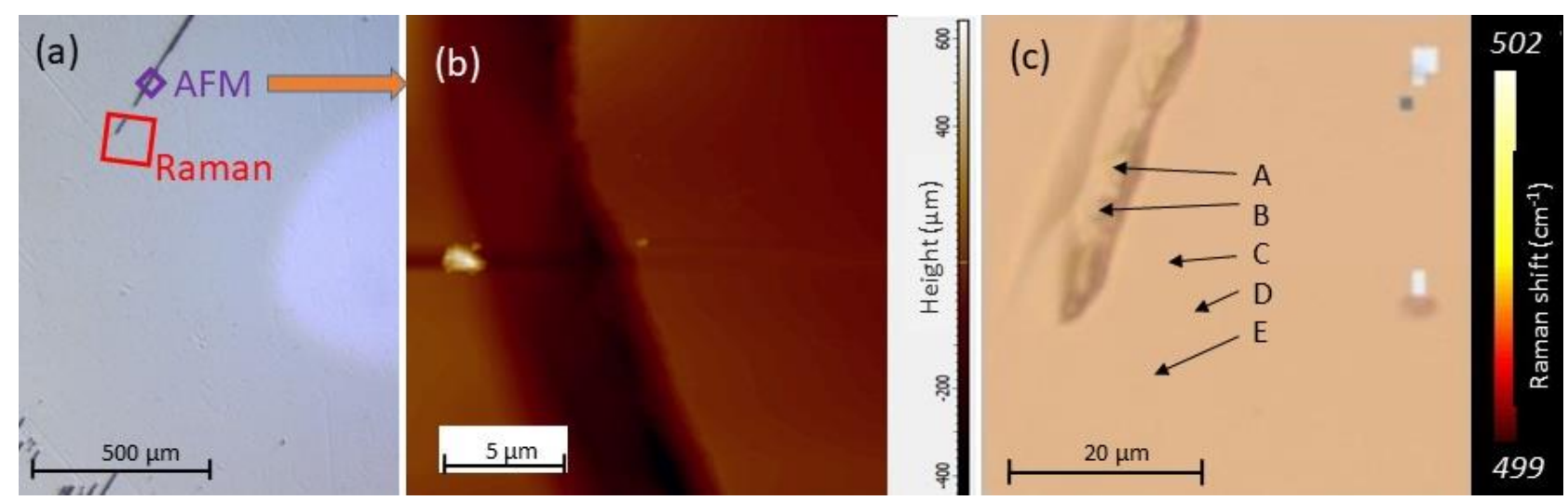

Figure 4 (a) Optical microscopy image of the (113) - oriented boron-doped diamond surface;

(b) AFM image of the diamond's surface of the area indicated by the violet square on the optical microscopy image; (c) Raman $500 \mathrm{~cm}^{-1}$ peak map of the area indicated by the red square in the optical microscopy 


\section{CONCLUSION}

In this work, we compared the surface morphology as observed by the optical microscopy, the atomic force microscopy images, and the boron incorporation as determined by Raman spectroscopy in MWPECVD homoepitaxial boron-doped diamond layers grown on substrates with different crystalline orientations. We observed clearly marked and characteristic surface morphologies and the boron incorporation on the different epitaxial layers. Although macroscopically flat, the (111) epitaxial layer shows an irregular roughness at the submicrometer scale and a small boron concentration inhomogeneity. The (100) epitaxial layer is covered by characteristic rectangular based pyramidal hillocks, and the boron incorporation is very inhomogeneous. In contrast with the (111) and (100) epitaxial layers, the boron-doped (113) homo-epitaxial layer exhibits a very smooth surface combined with homogeneous boron incorporation. On top of it, the (113) layer grows more swiftly, and the boron incorporation efficiency is the highest. These results are very promising for the development of vertical diamond power devices, which necessitates thick highly boron-doped templates.

\section{ACKNOWLEDGEMENTS}

This work was primarily financially supported by the Czech Science Foundation grant No. 20-11140S and by the Czech Technical University Student Grant No. SGS20/176/OHK3/3T/13. This work has also been supported by Operational Program Research, Development, and Education financed by European Structural and Investment Funds and the Czech Ministry of Education, Youth and Sports (Project No. SOLID21 - CZ.02.1.01/0.0/0.0/16_019/0000760).

\section{REFERENCES}

[1] ZHANG, B., WANG, S. A Survey of EMI Research in Power Electronics Systems with Wide-Bandgap Semiconductor Devices. IEEE J. of Emerging and Sel.Topics in Power Electronics. 2019, vol. 8, pp. 626-643.

[2] UMEZAWA, H. Recent advances in diamond power semiconductor devices. Materials Science in Semiconductor Processing. 2018, vol. 78, pp. 147-156.

[3] VOLPE, P. N. et al. Extreme dielectric strength in boron doped homoepitaxial diamond. Applied Physics Letters. 2010, vol. 97, p. 223501.

[4] TALLAIRE, A. et al. Growth of thick and heavily boron-doped (113)-oriented CVD diamond films. Diamond and Related Materials. 2016, vol. 66, pp. 61-66.

[5] MORTET, V. et al. Determination of atomic boron concentration in heavily boron-doped diamond by Raman spectroscopy. Diamond \& Related Materials 2019, vol. 93, pp. 54-58.

[6] WANG, X. et al. Surface Morphology of the Interface Junction of CVD Mosaic Single-Crystal Diamond. Material. 2020, vol. 13, p. 91.

[7] MORTET, V. et al. Insight into boron-doped diamond Raman spectra characteristic features. Carbon. 2017, vol. 115, pp. 279-284.

[8] BERNARD, M., DENEUVILLE, A., MUR, P. Non-destructive determination of the boron concentration of heavily doped metallic diamond thin films from Raman spectroscopy. Diamond and Related Materials. 2004, vol. 13, pp. 282-286.

[9] ACHATZ, P. et al. Isotopic substitution of boron and carbon in superconducting diamond epilayers grown by MPCVD. Diamond \& Related Materials. 2010, vol.19, pp. 814-817.

[10] SRIMONGKON, K. et al. Boron inhomogeneity of HPHT-grown single-crystal diamond substrates: Confocal microRaman mapping investigations. Diamond \& Related Materials. 2016, vol. 63, pp. 21-25.

[11] MORTET, V. et al. New perspectives for heavily boron-doped diamond Raman spectrum analysis. Carbon. 2020, vol. 168 pp. 319-327. 